\title{
Scaffold attachment factor B1: an intrinsic inhibitor of androgen receptor downregulated in prostate cancer
}

\author{
Haojie Huang ${ }^{1,2,3}$ \\ Asian Journal of Andrology (2013) 15, 703-704; doi:10.1038/aja.2013.116; Published online: 30 September 2013
}

$\mathrm{T}$ he androgen receptor (AR) is a pleiotrophic transcription factor that regulates expression of a large number of genes involved in many diverse cellular processes. The AR activation pathways have been studied extensively. However, the molecular mechanism and biological significance of AR inhibitory signals remain poorly understood. Mukhopadhyay et al. have now identified the nuclear matrix protein scaffold attachment factor B1 (SAFB1) as a novel AR corepressor. The authors found that SAFB1 physically associates with AR protein and inhibits AR transcriptional activity and androgen-sensitive gene expression. SAFB1 had no effect on chromatin occupancy of AR, but silencing of SAFB1 abolished recruitment MST1, a known AR repressor, at AR target loci. They also showed that SAFB1 interacts with EZH2, SUZ12 and EED, three core components of the Polycomb repressive complex 2 (PRC2), which catalyzes the gene repression histone modification $\mathrm{H} 3$ lysine 27 trimethylation (H3K27me3). The authors further showed that forced expression of SAFB1 increases H3K27me3 at AR target loci and this effect requires EZH2. Finally, the authors demonstrated that expression of SAFB1 is downregulated in human prostate cancer (PCa) specimens and SAFB1 knockdown results in an aggressive phenotype of PCa. These findings identify SAFB1 as an important node for integration of multiple inhibitory signals of $A R$, which represents a viable pathway for therapeutic intervention of PCa.

Androgens and the androgen receptor (AR) are important for growth and differentiation

${ }^{1}$ Department of Biochemistry and Molecular Biology, Mayo Clinic College of Medicine, Rochester, MN 55905, USA; ${ }^{2}$ Department of Urology, Mayo Clinic College of Medicine, Rochester, MN 55905, USA and ${ }^{3}$ Mayo Clinic Cancer Center, Mayo Clinic College of Medicine, Rochester, MN 55905, USA

Correspondence: Dr H Huang (huang.haojie@

mayo.edu) of the normal prostate gland. They are also the major factors required for development and progression of prostate cancer (PCa). ${ }^{1}$ Androgen deprivation therapy is the primary treatment option for patients with advanced/ disseminated PCa. Despite the initial therapeutic response, a majority of prostate cancers eventually evolve into a disease termed hormone refractory or castration-resistant $\mathrm{PCa}$ (CRPC), from which most patients eventually succumb. In virtually all cases, castrationresistant progression is accompanied by a rise in the level of prostate-specific antigen, a wellstudied AR transactivated gene, indicating that the $\mathrm{AR}$ is aberrantly activated under castration conditions. The importance of persistent AR signaling in CRPC is further demonstrated by the antitumor activity of next-generation androgen-AR axis inhibitors including abiraterone and enzalutamide. ${ }^{2,3}$ It is hence of importance to fully understand the mechanisms of aberrant activation of the AR in PCa.

Mukhopadhyay et al. ${ }^{4}$ found that androgen stimulation induces formation of an scaffold attachment factor B1 (SAFB1)/AR complex and that silencing of endogenous SAFB1 enhanced androgen-induced transactivation of AR. These findings are highly significant because they reveal a novel mechanism of 'checkpoint' that counterbalances the androgenic activation of the AR. To define the molecular mechanism of SAFB1 inhibition of the AR, Mukhopadhyay et al. ${ }^{5}$ examined if this effect of SAFB1 involves MST1, a known AR repressor. MST1 is a serine/threonine kinase, the homolog of Hippo that was first defined as a tumor suppressor gene in Drosophila. ${ }^{6}$ The evolutionally conserved MST1/Hippo pathway plays essential roles in regulation of cell proliferation and differentiation. ${ }^{6}$ Both MST1 and its downstream effector LATS2 have been shown to be negative regulators of AR function and $\mathrm{PCa}$ cell growth. ${ }^{5,7}$ Mukhopadhyay et al. ${ }^{4}$ showed that MST1 interacts with and phosphorylates SAFB1 in PCa cells. Chromatin immunoprecipitation and re-chromatin immunoprecipitation studies showed that SAFB1 and active MST1 are present in the same complex associated with the enhancer/promoters of $\mathrm{AR}$ target genes examined. While SAFB1 knockdown had no effect on AR occupancy on chromatin, it inhibited MST1 recruitment to $\mathrm{AR}$ target gene loci, indicating that SAFB1 functions downstream of MST1.

Because silencing of endogenous MST1 only partially attenuated SAFB1-mediated inhibition of AR activity, Mukhopadhyay et al. ${ }^{4}$ further explored the mechanism of inhibitory action of SAFB1. Histone H3K9, H3K27 and H4K20 are three known lysine methylation sites that are associated with transcriptional repression. The authors found that forced expression of SAFB1 increased H3 lysine 27 trimethylation ( $\mathrm{H} 3 \mathrm{~K} 27 \mathrm{me} 3)$ in the prostatespecific antigen promoter. Accordingly, they reported that EZH2, SUZ12 and EED, three major components of Polycomb repressive complex 2 (PRC2) complex that is responsible for $\mathrm{H} 3 \mathrm{~K} 27 \mathrm{me} 3,{ }^{8}$ form a protein complex with SAFB1 in PCa cells. Importantly, SAFB1induced increase in $\mathrm{H} 3 \mathrm{~K} 27 \mathrm{me} 3$ levels at the prostate-specific antigen locus was abolished in EZH2-knockdown cells. ${ }^{4}$ The authors further showed that all the core members of the PRC2 complex colocalize with SAFB1 at AR target gene promoters. ${ }^{4}$ These data suggest that the PRC2 complex and H3K27me3 are key effectors of SAFB1 inhibition of the AR.

In agreement with the data obtained by Mukhopadhyay et al., ${ }^{4}$ it has been shown by a global chromatin immunoprecipitation-seq analysis that the repressive histone mark $\mathrm{H} 3 \mathrm{~K} 27 \mathrm{me} 3$ is enriched around both ARinduced and repressed gene loci, although the enrichment seems higher at AR-repressed than activated loci. ${ }^{9}$ Moreover, androgen 


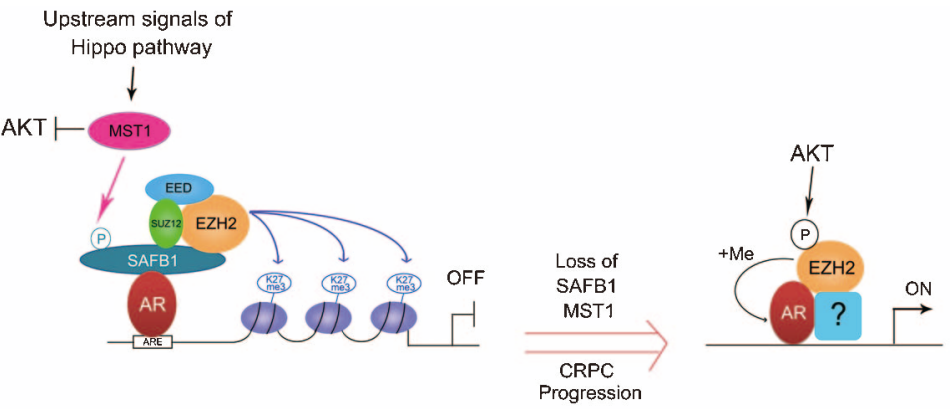

Figure 1 SAFB1 regulation of AR activity during PCa progression. Activated by its upstream signals, the serine/threonine kinase MST1 interacts with and phosphorylates SAFB1 in primary PCa. By working in concert with MST1 and the PRC2 complex, phosphorylated SAFB1 inhibits AR transcriptional activity, at least in part, by enhancing H3K27me3 at AR target gene loci. During castration-resistant progression of PCa, loss or reduced expression of SAFB1 and MST1 not only abolishes SAFB1 inhibition of AR-mediated transcription of classical AR regulated genes, but may also facilitate AKT phosphorylation-dependent AR-EZH2 interaction, thereby resulting in transcription reprograming in CRPC cells. AR, androgen receptor; ARE, androgen responsive element; CRPC, castration-resistant prostate cancer; H3K27me3, H3 lysine 27 trimethylation; PCa, prostate cancer; PRC2, Polycomb repressive complex 2; SAFB1, scaffold attachment factor B1.

stimulation increased H3K27me3 levels at both AR-induced and repressed genes. ${ }^{9}$ Given that little to no physical interaction was detected between AR and EZH2 in androgen-responsive cells, ${ }^{10}$ it is warranted to determine if SAFB1 functions as a scaffold to facilitate AR-EZH2 association and their functional interaction. It is also important to determine in the future if SAFB1 affects the H3K27me3 landscape genome-wide at the enhancer/promoters of classical AR activated genes.

The study performed by Mukhopadhyay et al. ${ }^{4}$ is highly relevant because their tissue microarray data showed that SAFB1 levels were significantly lower in metastases in comparison with both benign tissues and locally defined PCa. Although the authors did not show the data, they reported that the lowest levels of SAFB1 protein were detected in eight out of $13(>60 \%)$ of metastatic CRPC. Overexpression of EZH2 has been shown to be associated with castration-resistant progression of PCa. ${ }^{11}$ This observation is consistent with the finding that androgens induce repression of EZH2, and androgen withdrawal by castration upregulates EZH2 expression in animals. ${ }^{12}$ Intriguingly, it has been reported recently that EZH2 goes solo (independent of the PRC2 complex) to bind to and enhance AR transcriptional activity. ${ }^{13}$ It is worth noting that such a Polycomb-independent function of EZH2 only occurs in CRPC cells and that this effect is likely mediated by AKT-dependent phosphorylation of EZH2 at serine 21 and phosphorylation-dependent interaction between EZH2 and AR. ${ }^{13}$ Moreover, it has been shown previously that MST1 is a direct inhibitor of AKT and that reduced expression of MST1 expression correlates with $\mathrm{PCa}$ progression to the castration-resistant metastatic state. ${ }^{14}$ Based upon the reports and the findings demonstrated by Mukhopadhyay et al., ${ }^{4}$ we envisage a model where SAFB1 functions as a scaffold that enables to recruit PRC2 components into the AR transactivation complex and this function of SAFB1 is affected by MST1-mediated phosphorylation. Thus, SAFB1 acts as a novel AR corepressor by integrating signals from the MST1/Hippo and EZH2 pathways (Figure 1). During castration resistant progression of $\mathrm{PCa}$, however, loss or reduced expression of MST1 and SAFB1 may increase AKT-mediated phosphorylation of EZH2 at serine 21 and enhance the accessibility of EZH2 to AR, respectively, thereby promoting the shift from the PRC2/ H3K27me3-dependent AR inhibition mode to the Polycomb-independent but EZH2 dependent AR activation mode (Figure 1). Future experimental examination of this hypothesis is warranted, and findings from these investigations may not only shed new light on the AR inhibitory pathways, but also lead to identification of new targets for therapeutic intervention of CRPC.

1 van der Steen T, Tindall DJ, Huang H. Posttranslational modification of the androgen receptor in prostate cancer. Int J Mol Sci 2013; 14: 14833-59.

2 Scher HI, Fizazi K, Saad F, Taplin ME, Sternberg CN et al. Increased survival with enzalutamide in prostate cancer after chemotherapy. N Engl J Med 2012; 367: 1187-97.

3 de Bono JS, Logothetis CJ, Molina A, Fizazi K, North S et al. Abiraterone and increased survival in metastatic prostate cancer. N Engl J Med 2011; 364: 19952005.

4 Mukhopadhyay NK, Kim J, You S, Morello M, Hager $\mathrm{MH}$ et al. Scaffold attachment factor $\mathrm{B} 1$ regulates the androgen receptor in concert with the growth inhibitory kinase MST1 and the methyltransferase EZH2. Oncogene; e-pub ahead of print 29 July 2013; doi:10.1038/onc.2013.294.

5 Cinar B, Collak FK, Lopez D, Akgul S, Mukhopadhyay NK et al. MST1 is a multifunctional caspaseindependent inhibitor of androgenic signaling. Cancer Res 2011; 71: 4303-13.

6 Yu FX, Guan KL. The Hippo pathway: regulators and regulations. Genes Dev 2013; 27: 355-71.

7 Powzaniuk M, McElwee-Witmer S, Vogel RL, Hayami $\mathrm{T}$, Rutledge SJ et al. The LATS2/KPM tumor suppressor is a negative regulator of the androgen receptor. Mol Endocrinol 2004; 18: 2011-23.

8 Chen S, Bohrer LR, Rai AN, Pan Y, Gan L et al. Cyclindependent kinases regulate epigenetic gene silencing through phosphorylation of EZH2. Nat Cell Bio/2010; 12: 1108-14.

9 Zhao JC, Yu J, Runkle C, Wu L, Hu M et al. Cooperation between Polycomb and androgen receptor during oncogenic transformation. Genome Res 2012; 22: 322-31.

10 Grasso CS, Wu YM, Robinson DR, Cao X, Dhanasekaran SM et al. The mutational landscape of lethal castration-resistant prostate cancer. Nature 2012; 487: 239-43.

11 Varambally S, Dhanasekaran SM, Zhou M, Barrette $\mathrm{TR}$, Kumar-Sinha $\mathrm{C}$ et al. The polycomb group protein $\mathrm{EZH} 2$ is involved in progression of prostate cancer. Nature 2002; 419: 624-9.

12 Bohrer LR, Chen S, Hallstrom TC, Huang H. Androgens suppress EZH2 expression via retinoblastoma (RB) and p130-dependent pathways: a potential mechanism of androgen-refractory progression of prostate cancer. Endocrinology 2010; 151: 5136-45.

$13 \mathrm{Xu} \mathrm{K,} \mathrm{Wu} \mathrm{ZJ,} \mathrm{Groner} \mathrm{AC,} \mathrm{He} \mathrm{HH,} \mathrm{Cai} \mathrm{C} \mathrm{et} \mathrm{al.} \mathrm{EZH2}$ oncogenic activity in castration-resistant prostate cancer cells is Polycomb-independent. Science 2012; 338: 1465-9.

14 Cinar B, Fang PK, Lutchman M, Di Vizio D, Adam RM et al. The pro-apoptotic kinase Mst1 and its caspase cleavage products are direct inhibitors of Akt1. EMBO J 2007; 26: 4523-34. 